\section{$\underset{\substack{\text { hommes } \\ \text { \& migrations }}}{ }$}

\section{Hommes \& migrations}

Revue française de référence sur les dynamiques

migratoires

\section{8 | 2014}

Les Paris des migrants

\title{
Les travailleurs chinois en France pendant la Grande Guerre
}

\author{
Laurent Dornel
}

\section{OpenEdition \\ Journals}

Édition électronique

URL : http://journals.openedition.org/hommesmigrations/3026

DOI : 10.4000/hommesmigrations.3026

ISSN : 2262-3353

Éditeur

Musée national de l'histoire de l'immigration

Édition imprimée

Date de publication : 1 octobre 2014

Pagination : 174-178

ISBN : 978-2-919040-29-2

ISSN : $1142-852 X$

Référence électronique

Laurent Dornel, « Les travailleurs chinois en France pendant la Grande Guerre », Hommes \& migrations [En ligne], 1308 | 2014, mis en ligne le 26 juin 2015, consulté le 23 septembre 2020. URL : http:// journals.openedition.org/hommesmigrations/3026; DOI : https://doi.org/10.4000/ hommesmigrations.3026 


\section{CHRONIQUES DE GUERRE}

\section{LES TRAVAILLEURS CHINOIS EN FRANCE PENDANT LA GRANDE GUERRE}

LAURENT DORNEL, maître de conférences en histoire contemporaine, université de $\mathrm{Pau}$

Jusqu'en 1917, la Chine fut neutre, quoique en réalité concernée par la guerre européenne. En effet, on comptait alors près d'une trentaine de zones de concessions étrangères ou territoires à bail réunissant de très nombreux belligérants. Si l'envoi de soldats fut très tôt écarté, en revanche, Britanniques et Français - pour qui la Chine était traditionnellement un réservoir de main-d'œuvre-y puisèrent des travailleurs. C'est ainsi que 100000 Chinois furent recrutés par les Britanniques, près de 40000 par les Français'. Tous œuvrèrent exclusivement en France, les premiers aux tâches logistiques à l'arrière des troupes anglaises, les seconds plus généralement au service de l'économie de guerre. Ce fut pour tous une expérience souvent difficile mais encore mal connue tant le souvenir en a disparu des mémoires collectives.

\section{Des Chinois au service de l'économie de guerre française}

Du côté français, l'essentiel des travailleurs des chinois fut recrute dans le cadre de la mission Truptil, du nom d'un militaire français. Les discussions durèrent plusieurs mois et furent finalement entérinées par les deux gouvernements français et chinois. Pour procéder au recrutement des travailleurs, Truptil conclut un accord avec un intermédiaire chinois qui se constitua alors, le syndicat Huimin. Au terme d'un long périple, un premier contingent de presque 1700 travailleurs chinois arriva à Marseille le 24 août 1916. Du côté britannique, les recrutements furent plus tardifs en raison de l'hostilité du gouvernement comme des syndicats. Les Chinois, acheminés jusqu'au Havre, étaient ensuite affectés à des dépôts parfois immenses, à l'arrière des troupes. Ils remplirent presque exclusivement des tâches logistiques (déchargement des munitions, réparation des routes et des tranchées, désobusage à la fin du conflit...).

L'immense majorité de ces travailleurs - recrutés surtout dans le Nord - étaient des paysans jeunes, pauvres et illettrés. Les contrats étaient de trois ans avec la partie britannique et de cinq ans avec la partie française. Une avance était versée au moment de l'embarquement et une prime d'engagement versée à la famille. Côté français, et pour des tâches similaires, le contrat garantissait en principe l'égalité des salaires entre ouvriers chinois et nationaux. Dans l'ensemble, les conditions de travail et de vie des travailleurs sous autorité britannique furent souvent plus difficiles. Ajoutons enfin que 10000 Chinois "français" furent prêtés à l'armée américaine.

En arrivant à Marseille, les travailleurs étaient regroupés dans un vaste dépôt où ils subissaient une seconde visite médicale et ils étaient imma- 
triculés avant d'être acheminés vers des groupements régionaux où, enfin, on leur affectait un emploi. Ils furent employés en groupes plus ou moins importants ( 25 au minimum) et rassemblés en groupements de 200 à 250 hommes environ sur l'ensemble du territoire métropolitain. Les circulaires officielles stipulaient qu'ils ne devaient pas être mélangés à d'autres "races". Affectés à des travaux très variables : creusement et entretien des tranchées, réparation des voies de communication (routes, chemins de fer), déchargement et transport de munitions, ils furent également présents sur les ports (comme dockers), dans les usines et dans les mines.

L'une des caractéristiques de cette main-d'œuvre, c'est qu'elle était à peu près totalement inconnue en France avant la guerre. Elle fut donc dans un premier temps perçue au prisme des représentations que l'on se faisait alors des Chinois en France.

\section{Une main-d'œuvre "exotique" dépréciée par les stéréotypes}

Dans les représentations courantes, qu'elles fussent populaires on non, le terme "Chinois" sonnait souvent de façon dépréciative voire péjorative. Ainsi, à "Chinois", peut-on lire dans Le Petit Littré : "Se dit, en moquerie, de quelqu'un qui par sa tournure de corps ou d'esprit a quelque chose de burlesque ou de désagréable." Au stéréotype du casse-tête chinois, s'ajoutent ceux de la fourberie, de la férocité et de la cruauté extrême. À la fin du $X I X{ }^{\circledR}$ siècle et au début du siècle suivant, toute une littérature ${ }^{2}$ avait fait son miel de ces stéréotypes, auxquels s'ajoutaient des développements sur le "grouillement" ou la saleté, le tout repris dans le mythe de l'invasion ou du péril “jaune”.

Bien des questions se posèrent aux autorités françaises quant à ces travailleurs chinois : comment allait-on les prendre en charge ? Quels travaux allait-on leur confier ? Comment réglerait-on le délicat problème de la langue? Quelles relations souhaitait-on leur permettre avec les Français ? Fallait-il les autoriser à fréquenter les travailleurs coloniaux, c'est-à-dire les Indochinois, les NordAfricains ou encore les Malgaches employés par milliers dans les usines ?

Alors, pour accompagner leur arrivée sur le marché du travail, des Instructions et des Notices furent rédigées, notamment par le Service de l'organisation des travailleurs coloniaux (SOTC) dont les Chinois dépendaient, service placé sous l'autorité de la Direction des troupes coloniales et situé au ministère de la Guerre ${ }^{3}$. Les Chinois, assimilés de fait aux ouvriers nord-africains ou indochinois, y étaient décrits ainsi : "En général grands et vigoureux, ils supportent également bien le froid et la chaleur. Ils se montrent, le plus souvent, sobres et laborieux. Agriculteurs passionnés, ils cultivent la terre avec un soin extrême. Ils sont d'autre part très habiles dans le commerce vers lequel les attire leur tournure d'esprit. Aptes à devenir de très bons ouvriers, ils possèdent des facultés particulières d'assimilation et d'imitation qui leur permettent de se perfectionner rapidement dans leur métier." Dans les documents officiels comme dans la presse, les travailleurs chinois étaient désignés comme des "indigènes" ou des "exotiques", termes qui renvoyaient à l'imaginaire et au monde coloniaux. Ils apparaissaient parfois aussi sous l'appellation de "coolies", ce qui les ravalait au rang d'une force de travail. Mais on trouvait d'autres désignations : "Orientaux”, “Célestes", “Asiatiques”. Alors qu'ils étaient en théorie des civils, dans les faits, et en dépit des rappels officiels, ils furent militarisés : ils vivaient dans des cantonnements sous la surveillance de cadres militaires et ils étaient conduits vers leur lieu de travail sous bonne escorte. II fallait à la fois éviter autant que possible le contact avec la population française et pouvoir surveiller et contrôler cette main-d'œuvre mal connue, potentiellement turbulente. Ces 


\section{CHRONIQUES DE GUERRE}

contraintes furent bien souvent rejetées par les intéressés qui avaient par ailleurs une réputation d'indiscipline. Dès novembre 1916, à la poudrerie nationale d'Oissel, eut lieu une émeute d'ouvriers chinois. Au cours des mois et des années suivants, les troubles liés à la présence des Chinois ne cessèrent pas : à plusieurs reprises en 1916, 1917 et 1918, les travailleurs chinois firent grève, voire provoquèrent des émeutes (par exemple, en 1918 : poudrerie de Saint-Louis-du-Rhône, Ambronay, Société des grands travaux de Marseille de Beaumont-Monteux ${ }^{4}$ ), ce qui ne fit qu'accentuer le contrôle disciplinaire dont ils étaient l’objet.

\section{Pour les travailleurs chinois, une expérience souvent difficile}

Il faut avant tout rappeler les réticences initiales à l'introduction (en France comme en Angleterre) des travailleurs chinois : lors du Conseil des ministres du 30 mars 1915, Alexandre Ribot - alors ministre des Finances - s'y était opposé, soulignant que "tous les pays où les Chinois sont venus ont été infestés de cette race quelques années plus tard", tout comme Gaston Doumergue (ministre des Colonies), qui déclarait avec violence que "les Colonies ne veulent pas de travailleurs chinois s". De son côté, en septembre 1916, Léon Jouhaux, pour la Confédération générale du travail (CGT), protesta auprès d'Albert Métin, ministre du Travail, "contre l'introduction sur notre marché du travail, de 20000 nouveaux Chinois"." Ce même Métin, en mars 1916, craignait d'ailleurs que le recrutement n'introduise un grand nombre d'éléments indésirables: "Dans beaucoup de pays, les travailleurs chinois ont donné lieu à des plaintes très vives de la part des populations, on leur reproche leur saleté, leur peu de souci des précautions élémentaires d'hygiène?." Dans bien des cas, cette hostilité de principe plus ou moins marquée a pu s'accompagner de pratiques combinant négligence, incompréhension rédhibitoire, vexations, voire violences.

De nombreuses plaintes relatives à la nourriture, souvent relayées par les cadres français, témoignent d'une réelle et fréquente négligence envers les ouvriers chinois auxquels on servit plus d'une fois de la viande de cheval considérée en Chine comme non comestible. Bien d'autres formes de négligences les touchèrent : à maintes reprises, on leur distribua des vêtements et des souliers fort usés.

Bien des témoignages soulignent par ailleurs le refus fréquent d'employer les Chinois, bien que les motifs n'en soient généralement pas explicités. Ainsi le chef du Service central d'exploitation des ports de commerce de Cherbourg soulignait en septembre 1918 "la répugnance invincible que montrent certains usagers du port à se servir de la main-d'cuure chinoise ${ }^{8 "}$. De l'hostilité diffuse aux mauvais traitements, le pas fut parfois franchi. Les mauvais traitements furent tels qu'en mars 1918, le SOTC estima nécessaire de rédiger une circulaire édictant "'interdiction de frapper en aucun cas les travailleurs chinois et coloniaux ${ }^{9}$ ".

\section{La multiplication des incidents avec la population française}

Avec les populations françaises, les relations ne furent pas simples non plus, oscillant entre fascination et rejet. Des dizaines de rixes opposèrent les travailleurs chinois aux populations locales, aux soldats français ou encore à d'autres tra- 
vailleurs étrangers ou coloniaux. En janvier 1917, deux travailleurs chinois meurent après une rixe contre des Arabes, à la poudrerie de Bassens. Quelques jours plus tard, à la poudrerie de Bergerac, 500 Chinois affrontent 250 Algériens : on relève un mort (un Chinois) et une soixantaine de blessés. Si pour les incidents de Bassens et de Bergerac, la responsabilité des Chinois pouvait éventuellement être engagée, de nombreux autres incidents ${ }^{10}$ témoignèrent d'une hostilité fréquente envers les travailleurs chinois. Ainsi, en mars 1918, le SOTC évoquait "les regrettables incidents survenus dans la 7 région où des militaires permissionnaires poussés par certains éléments de la population civile ont tiré sur des travailleurs chinois"1." Quelques semaines plus tard, en mai, une rixe éclata entre Chinois et artilleurs et automobilistes français à Laronxe (Meurthe-etMoselle). "Cet incident regrettable, écrit le capitaine de Nouaillan, est la suite des plaisanteries et railleries que nos hommes s'obstinaient malgré les défenses et les conseils de leurs chefs à faire au passage des Chinois ${ }^{12}$." Pendant la guerre, populations locales ou autorités administratives accusèrent les travailleurs chinois d'“indécence". En mars 1918, le maire de Moncel-les-Lunéville se plaignit que "ces gens sont malpropres, ils prennent les cantonnements pour des écuries et des fosses d'aisance. D'autre part, ils ne craignent pas de s'exhiber tout nus'"

Les archives témoignent d'une étonnante capacité des Chinois à sortir des cantonnements auxquels ils étaient assignés. Si elles conservent les plaintes, elles laissent également deviner des relations plus étroites. Des relations commerciales d'abord, puisque dans quelques cas elles révèlent que les travailleurs chinois trafiquaient avec les populations françaises ou les travailleurs coloniaux, leur revendant par exemple le pain ou le tabac qu'on leur fournissait. Et des relations sexuelles aussi, dont on sait qu'elles ne furent pas rares. Ainsi, en février 1917, le député Pascal Ceccaldi, chargé de mission pour l'examen des conditions de travail de la main-d'œuvre coloniale, notait à propos des 401 Chinois employés à la poudrerie de Saint-Chamas : "État sanitaire : excellent. Le climat convient admirablement aux Chinois. Quelques maladies d'origine sexuelle. Les Chinois paraissent ne pas être trop privés du sexe faible ${ }^{14}$."

Les incidents avec les travailleurs chinois se multiplièrent à l'occasion de leur démobilisation, notamment à l'automne 1919, et tout particulièrement dans le nord et l'est de la France, au point de faire naître une quasi-psychose. Pétain, en tournée d'inspection dans la région de Lens, écrivit à Clemenceau pour évoquer "les graves inconvénients résultant de la présence dans cette région de travailleurs chinois (...). Ils constituent pour la région une cause permanente d'insécurité et de malaise. Les populations sont unanimes à demander leur éloignementis". Ces incidents, qui concernaient fréquemment les ouvriers chinois sous contrôle britannique, finirent par provoquer des tensions entre la France et l'Angleterre. Le rapatriement des travailleurs chinois sous autorité française fut plus lent : après avoir été employés au "nettoyage" des zones de combats (désobusage, exhumation des cadavres, etc.), ils participèrent à la reconstruction de l'après-guerre. Le dernier rapatriement organisé par le gouvernement français eut lieu en février 1922.

\section{Un épisode mieux connu}

Sur les quelque 40000 Chinois acheminés en France, 2000 environ décidèrent d'y demeurer, bénéficiant d'un contrat d'embauche (mines de La Machine, Renault...). À la fin des années 1920, il en restait autour d'un millier, souvent mariés à des Françaises. Au cours des années 1920, ils 


\section{CHRONIQUES DE GUERRE}

avaient été rejoints par les étudiants du "mouvement travail études" (Zhou Enlai, Deng Xiaoping, Chen Yi Li Lisan, Nie Rongzhen). On ne connaît pas exactement le nombre de ceux qui jamais ne revinrent, touchés notamment par l'épidémie de grippe espagnole. Du côté français, en tenant compte des victimes des torpillages, quelque 1500 Chinois auraient péri ; du côté britannique, autour de 2000.

Avec ceux de ces étudiants qui sont restés, les travailleurs de la Grande Guerre ont formé la première communauté chinoise de France, située dans un premier temps autour de la gare de Lyon, puis dans le quartier des Arts-et-Métiers. Une première plaque commémorative a été apposée place Maurice-Denis près de la gare de Lyon en 1988. Dix ans plus tard, dans le jardin Baudricourt $\left.(X I I)^{e}\right)$, a été érigée une sorte de stèle à la mémoire de ces travailleurs, à tort considérés comme des "combattants morts pour la France". Voilà en tout cas le signe d'un réveil mémoriel que l'on constate également en Chine. L'historiographie de la Chine communiste d'après 1949 avait considéré le recrutement des travailleurs chinois pendant la Première Guerre mondiale comme un épisode mineur et honteux appartenant à la longue histoire de l'exploitation de la Chine par les Occidentaux, en faisant un non-objet d'étude. Désormais, l'envoi de 140000 travailleurs en France est au contraire un objet d'histoire, comme en témoigne un nombre croissant de publications. 